\title{
Personalized Multimedia Integration for the Heterogeneous Museum Systems Using the Ontology Mapping Approach
}

\author{
Ngamnij Arch-int, Chatkamon Anontachai \\ Semantic Mining Information Integration Laboratory (SMIL), Computer Science Department, Science Faculty, Khan Kaen University, \\ Khan Kaen, Thailand. \\ Email: ngamnij@kku.ac.th, chatkamon.a@gmail.com
}

Received October $3^{\text {rd }}, 2012$; revised November $12^{\text {th }}, 2012$; accepted November $22^{\text {nd }}, 2012$

\begin{abstract}
Presently, many museums have developed their own multimedia information systems to store the artifacts and other objects of scientific, artistic, cultural or historical interest into the digital resources and make them available for public viewing on the Web. However, searching for the multimedia information is still not relevant to the user requirement, and the system does not provide meaningful information. This research work proposes the personalized multimedia integration system for museums based on ontology which is a core component of the Semantic Web technology. The multimedia information for each resource has been expressed in the Web Ontology Language (OWL). The research also resolved the problem of information integration by proposing the ontology mapping technique to cope with the semantic conflicts and structural conflicts via the OWL properties. Then the ontology storing users' interest was designed which matched the museum's ontology so that retrieval of multimedia information is meaningful and direct to the users' needs.
\end{abstract}

Keywords: Ontology Mapping; Personalized Multimedia Integration; Heterogeneous Museum Systems; Semantic Web

\section{Introduction}

Nowadays, many museums have developed their own multimedia information systems to store the artifacts and other objects of scientific, artistic, cultural or historical interest into the digital resources and make them available for public viewing on the Web. However, each multimedia information system can be developed or procure independently that differs from place to place. As a result, the systems as a whole have become heterogeneous and dependent on a variety of applications or database management systems. This heterogeneity has led to the following problems: 1) Each museum has its own information storing format or structure. For instance, Museum 1 names its object information table as "Object", whereas Museum 2 names the table as "Resource". Museum 1 stores creator's or object inventor's information in a creator table. Museum 2 stores its creator's information as an attribute in another table, which may make it difficult to find relationships between information sources; 2) Retrieval of information from each source is on the most part of keyword-based. The system still cannot retrieve information meaningfully by applying words that have similar meaning; 3 ) The system cannot directly re- trieve what the user needs if the user does not specify his or her requirement each time he is searching. For example, a user who prefers a video file should specify every time that he wants the video file.

In this paper, we present the ontology-based personalized multimedia integration architecture of a museum system which is a major component of the Semantic Web Technology [1]. The multimedia information structure from each source is extracted into the ontology expressed in Web Ontology Language (OWL) [2]. The local ontology from each source is integrated through the ontology mapping process to form the ontology-based personalized multimedia domain. This research aims to resolve the semantic and structural conflicts occurred during the ontology mapping process. To resolve the semantic conflicts, the research employs the principle of semantic similarity measurement through the WordNet [3] database. The research also employs the OWL properties to resolve the semantic conflicts, as well as the structural conflicts.

In addition, to obtain the results in accordance with the user's interest and requirement, the research transforms the personalized user profile into an ontological model. 
The user information stored in the user profile ontology can be used as a criterion to query the museum's information ontology so as to achieve efficient information retrieval that is mostly direct to the user's interests.

\section{Literature Reviews}

\subsection{Semantic Web Technology}

The Semantic Web technology enhances the capability of the present day Web technology to enable computer or software agent to understand Web information (machine understandable) which corresponds to human's understanding. Then the information can be further processed and managed by computer efficiently. Development of Semantic Web employs the ontology as an important component. The ontology defines a common vocabulary explicitly without any ambiguity so that both human and computer or software agent can understand and share information in a domain. One may consider to use ontology as an unified knowledge model for knowledge representation and vocabularies [4]. Hence, the semantic conflicts of information can be solved and the computer or software agent is able to search for the synonymous terms with similar meaning. Generally, ontology consists of classes or concepts, which in turn comprise groups of things called instances which have the same properties. Classes and instances can create relationships between classes or between instances. The relationship between classes can be called the subsumption hierarchy, whereby a general class (or superclass) subsumes more specific classes (or subclasses). The subsumption hierarchy is used to store properties at the level of generality and automatically provide them to the lower level of specific concepts through the inheritance mechanism. In addition, there is a general relationship that relies on properties as the connector. The property that links relationships between classes or between instances are called the ObjectProperty, whereas the property that connects classes or instances with literal is called the Data Type Property, which is the property used to describe each instance or class characteristic. Creating machine understandable ontology for computer or software agent requires transformation of ontology structure into a language form such as the Resource Description Framework (RDF/RDF Schema) $[5,6]$ and Web Ontology Language (OWL). OWL has RDF/S as its sublanguage, but adding more advanced constructs to describe semantics of RDF that enables the computer to understand the information meaning more than RDF/S. Moreover, in information search and retrieval from ontology, many more languages have been developed, for example, RQL [7], RDQL [8], SPARQL [9], OWL-QL [10], etc. This research relied on OWL to express the ontology structure and used SPARQL for searching and retrieving information from the ontology.

\subsection{DCMI-Dublin Core Metadata Initiative [11]}

The DC is the specialized metadata vocabulary defined by the Dublin Core Metadata Initiative (DCMI). The DCMI is an organization dedicated to promoting the widespread adoption of interoperable metadata standards for describing a wide range of networked resources. The DC consists of a set of predefined properties for describing digital resources unambiguously. The DC standard encompasses two levels: Simple and Qualified. Simple DC [12] (see Figure 1) comprises fifteen standard elements, for example: title, creator, subject, description, publisher, and so on; whereas Qualified DC [13] employs additional qualifiers called "Dublin Core Qualifiers" (DCQ) to further refine the meaning of a resource, for example: an element abstract is defined as an alternative qualifier to refine the description element. The simple DC usually uses prefix $d c$ as "http://purl.org/dc/elements/1.1/" namespace to annotate each standard element, whereas qualified DC usually uses prefix dcterms as "http://purl.org/dc/terms/" namespace. For example, the dc:title is used as a name given to the resource. Use of Dublin Core metadata enables resource owner to define explicit resource terms without ambiguity and lessen the problem of sharing and exchanging resource data between systems. A complete description for the DC metadata can be found in [11].

\subsection{Related Works}

Many research studies attempted to solve the problem of information integration from heterogeneous data sources. A number of studies [14-17] aimed at solving problems in multiplicity of information structures both in the databases and Web bases, by using ontology as an assistant mechanism for representing the information structure and mapping information from different systems which have

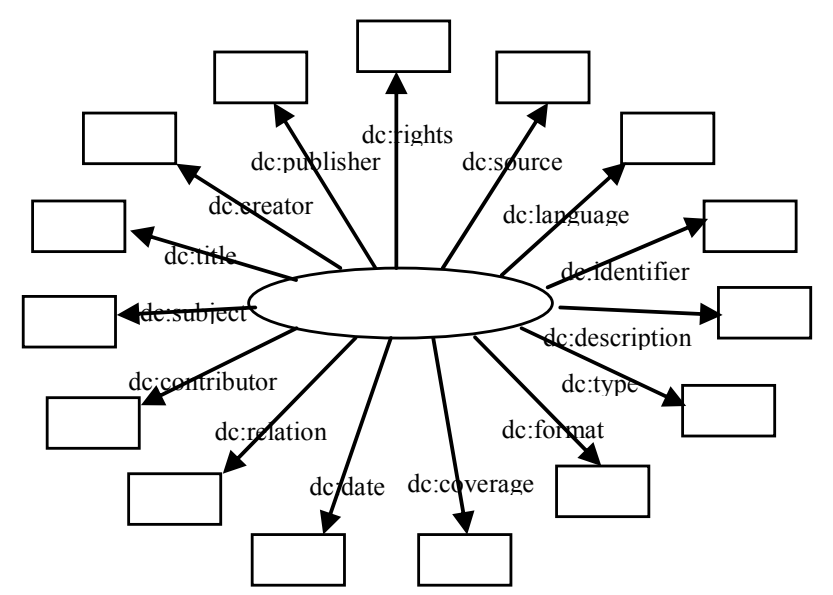

Figure 1. The simple DC standard [11]. 
structures and names discrepancies. This makes the information meaning understandable, and relationships between the information are correlated. The integration of information from multiple sources has to cope with the problems both in terms of structure and semantic conflicts. A lot of research works, for example [18-21] tried to solve the problem via ontology mapping [22] by applying various tools developed for the mapping or by relying on the WordNet and OWL properties to solve the problems. In the context of ontology personalization, D.-N. Chen, and Y.-C. Chiang [23] integrated ontology and collaborative filtering to design a system to provide information recommendation service. The system collects the information of the users and could learn the preferences of every user and those preferences in common which could be recommended to the users. X. Aimé, F. Furst, P. Kuntz and F. Trichet [24] provided the similarity measure dedicated to the personalization of a Domain Ontology by mainly adapting the content of an ontology to its context of use. An approach aims at talking about several parameters such as culture, educational background and emotional state to reflect the relevance users of ontologies perceive on the subclass hierarchies and to what extent the terms associated to the concepts are representative. Some other research studies $[25,26]$ emphasized on solving the integration of museum information from heterogeneous sources. However, most research works have not specifically solved structural conflicts and information retrieval does not really convey meaning directly according to the individual's interest.

\section{Research Methodology}

\subsection{System Architecture Design}

This research designed the system architecture consisting of three layers as shown in Figure 2, i.e., Resource Layer, Mediator Layer and Presentation Layer. Each layer has been designed with the following details:

\subsubsection{Resource Layer}

The resource layer is the layer in which each museum stores its multimedia data. This layer consists of the following components:

1) Database-This refers to the museum database for storing the multimedia digital resources and the personalized user profile.

2) Wrapper-The transforming of information stored in the relational database to the ontology expressed in OWL.

3) Local Ontology-Result of information transformation from the Wrapper module. The local ontology extraction consists of the personalized user profile ontology for storing users' interests and museum ontology for storing the multimedia information. The extracted local

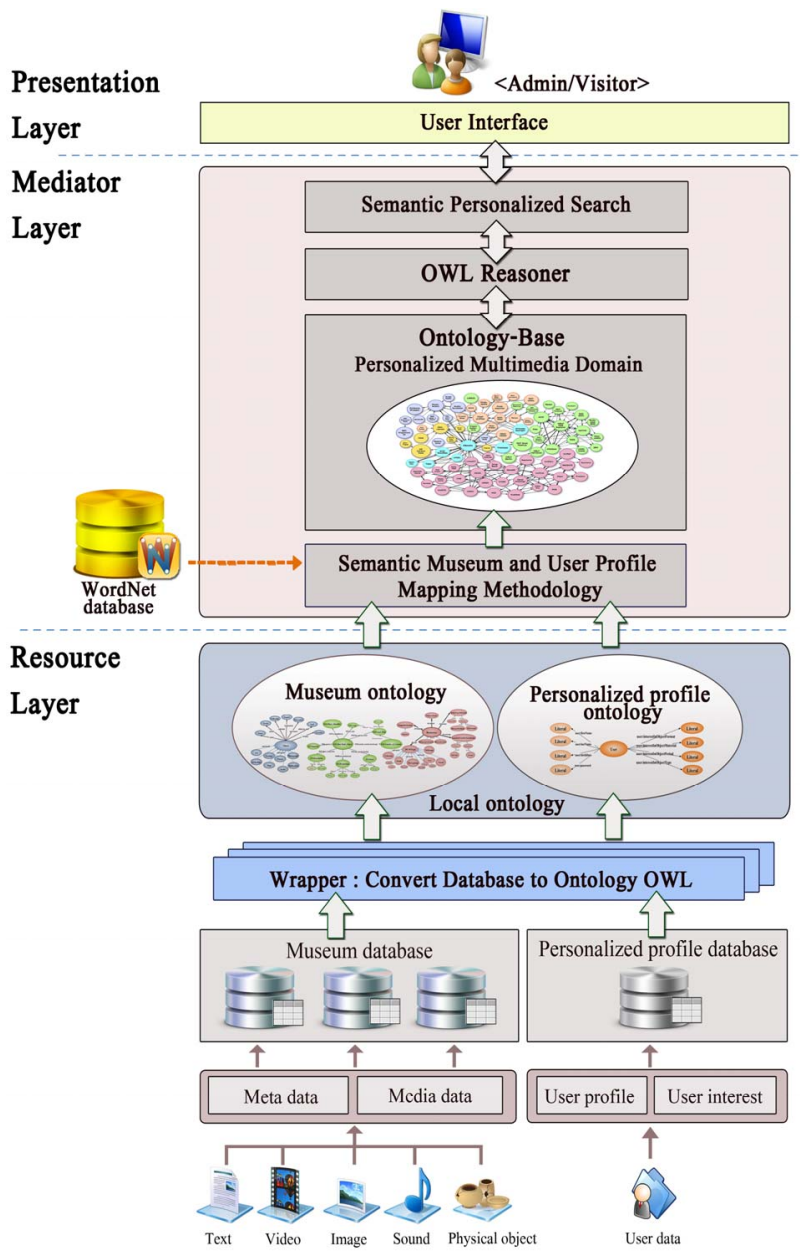

Figure 2. The system architecture design.

ontologies are expressed in OWL which enables machineunderstandable and semantic retrieval.

\subsubsection{Mediator Layer}

This layer mediates the information retrieval and integration system. The mediator layer is composed of the following operating components:

1) Semantic Personalized Search receives command from the user interface in the Presentation Layer and retrieves information from the mediator ontology according to user's condition.

2) Ontology-Based Personalized Multimedia Domain is a system's mediator ontology built from mapping of the museum local ontologies. This ontology is used as the main component for retrieving media information of the museum accurate to user's interest.

3) Semantic Museum Ontology Mapping is the module for mapping of the local museum ontologies from multiple museum resources. The semantic conflicts and structural conflicts arise during the ontology mapping process. To solve the semantic conflicts, this module employs Wordnet database to calculate the similarity value be- 
tween the concepts pair and the properties pair. The relation is built through OWL properties such as owl:equivalueClass and owl:equivalentProperty. To cope with the structural conflict, the solution relies on other properties of OWL such as owl:Restriction, owl:onProperty, and owl:someValuesFrom in bridging the different ontologies with structural conflict. The outcome of ontology conflict solution leads to the Ontology-Based Personalized Multimedia Domain.

4) Word Net database assists in locating semantic similarity of classes or properties between local ontologies which will be integrated to build a mediator ontology of the system.

5) OWL Reasoner is a tool providing various reasoning services for OWL documents, such as OWL species, consistency checking, satisfiability, and entailment test. The research employs the Pellet reasoner [27] for semantic information retrieval of the system's mediator ontology. The reasoner enables the computer to retrieve meaningful information and infer new knowledge stored in the mediator ontology to obtain deep relationship information not visible to general users.

\subsubsection{Presentation Layer}

This layer provides the user interface to receive registration data of users and record in the personalized profile database. The layer also receives retrieval command and conditions from the user, and then exhibits results of information retrieval from ontology and arrange appropriate format for the user.

\subsection{Ontology Design}

The museum information resources for research experiment were derived from two museum systems with dif- ferent database structures. The information from each system's database was extracted into ontology structure via the wrapper module. This research applied the research work [28] and the tools given in [29] for extracting information from database structure into ontology structure expressed in OWL. For this section, example of ontologies built from two sources of museum and a user profile ontology built from the personalized profile database will be shown.

The museum ontology of Museum 1 (see Figure 3) is the ontology extracted from the database schema of the Museum 1's system. The ontology structure is depicted as a graph consisting of classes, relationships between classes in the form of subsumption hierarchy, and the properties called the ObjectProperty such as status, period, and collection. These properties have been designed to have an object class as a domain, and the superclass properties can be inherited to different child classes.

1) The museum ontology of Museum 2 (see Figure 4) is the ontology extracted from the database schema of the Museum 2's system. This ontology employs the Dublin Core Metadata Element Set-DCMES to describe information, such as dc:title to describe the resource title, $d c:$ format to describe the resource format and dc:language to describe the resource language.

2) The museum ontology of Museum 2 (see Figure 4) is the ontology extracted from the database schema of the Museum 2's system. This ontology employs the Dublin Core Metadata Element Set-DCMES to describe information, such as dc:title to describe the resource title, $d c$ :format to describe the resource format and dc:language to describe the resource language.

3) User Profile Ontology, as shown in Figure 5, stores personal information and information about interest of users. This includes period, material, category, and for

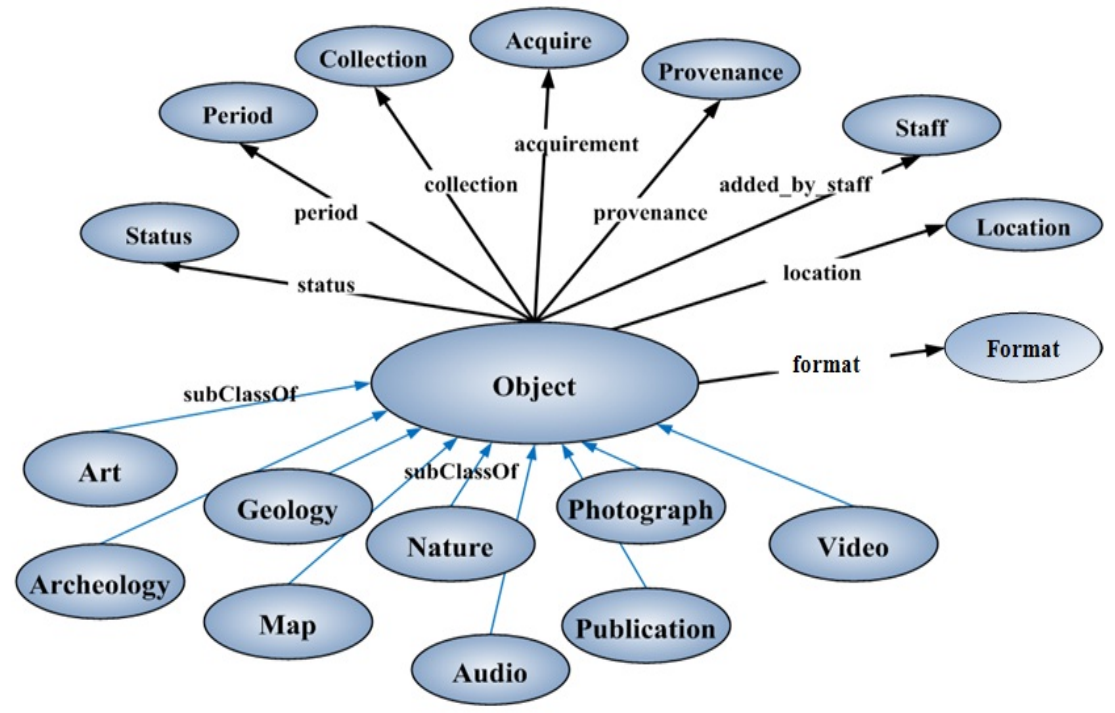

Figure 3. Ontology structure for storing data of Museum 1. 


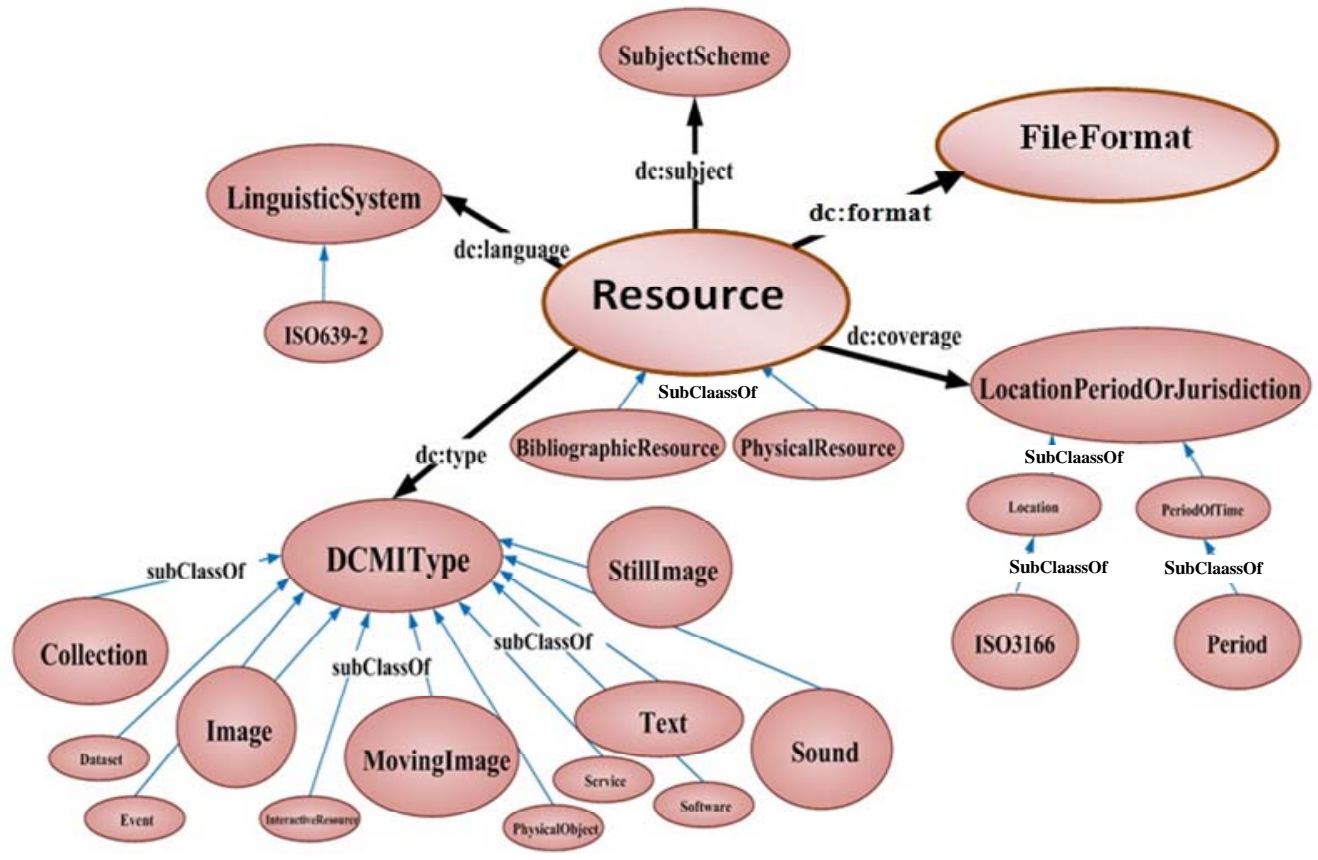

Figure 4. Ontology structure for storing data of Museum 2.

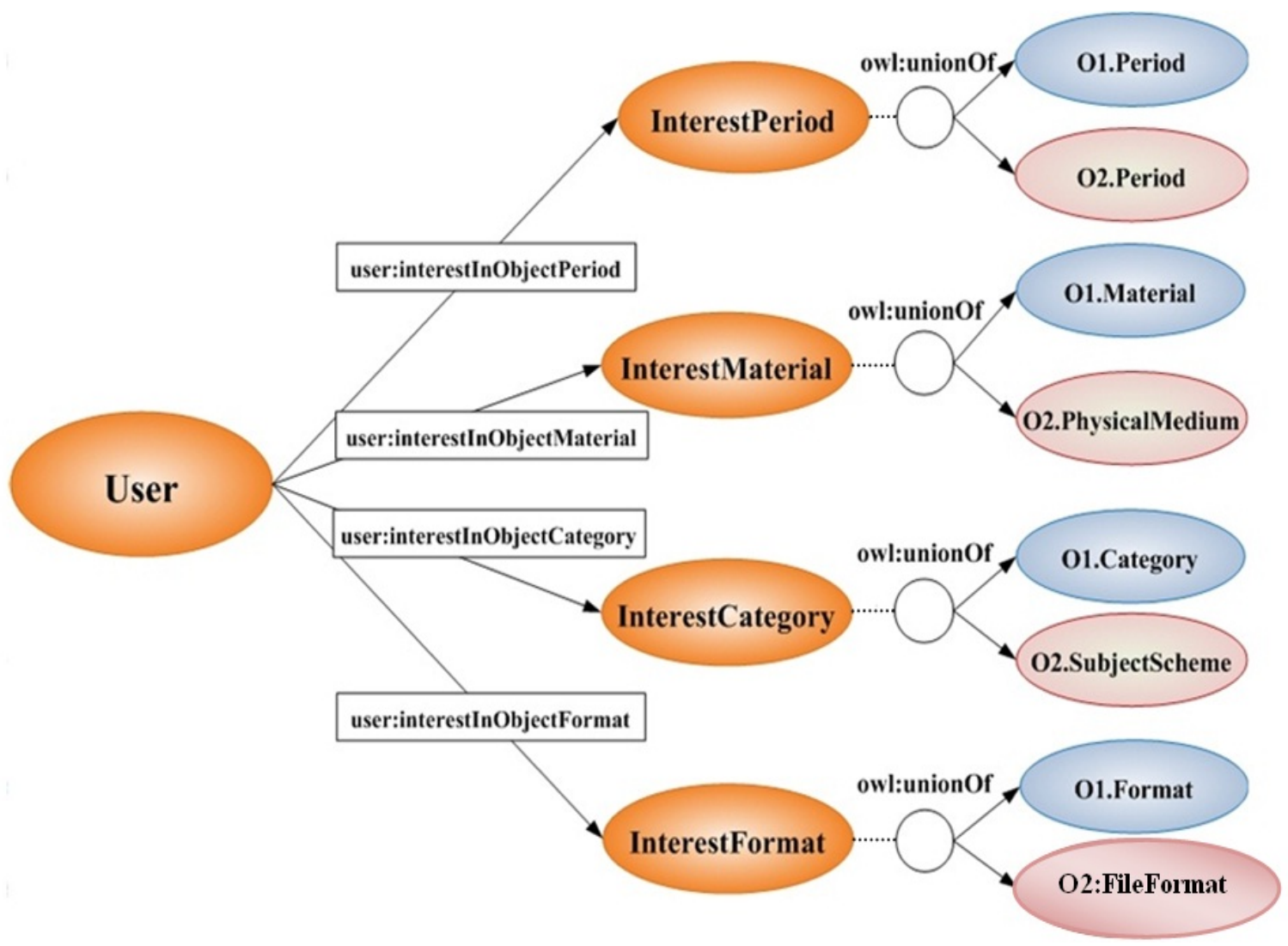

Figure 5. The user profile ontology.

mat of the objects which are stored into the user interest classes. The museum ontology classes associated to the user interest classes are combined through the owl:union
Of property, such that the instances retrieved from the museum ontology classes are shown for a user to specify his/her interest. 


\subsection{Ontology Integration}

Integration of data from ontologies that is derived from heterogeneous sources requires consideration of the heterogeneity problems. For this paper, we classified the heterogeneity problems into two main levels: the semantic heterogeneity and the structural heterogeneity as follows.

\subsubsection{Semantic Heterogeneity}

Occurs when there is a disagreement on the meaning, interpretation, or intended use of the same or related data. The semantic heterogeneity can be classified into various types, such as, semantic conflicts, property value conflicts, scaling conflicts, and so on. This paper focuses on semantic conflicts as described below:

Semantic conflicts, are concerned with the semantically equivalent classes or properties defined by different names. To solve the semantic conflicts, the concepts (classes or properties) pair from different ontologies is compared and computed the semantic similarity value based on the similarity value equation. In this research, the equation proposed by Wu and Palmer (wup) [30] was selected because it was designed on the basis of the WordNet to measure semantic similarity. The semantic similarity assessment is achieved by terming the compared words "concepts" as in the following Equation (1).

$$
\operatorname{Sim}_{\text {wup }}\left(c_{1}, c_{2}\right)=\frac{2 \times \operatorname{depth}\left(\operatorname{lcs}\left(c_{1}, c_{2}\right)\right)}{\operatorname{depth}\left(c_{1}\right)+\operatorname{depth}\left(c_{2}\right)}
$$

where depth is the distance from the concept node to the root of the hierarchy, and $\operatorname{lcs}\left(c_{1}, c_{2}\right)$ is the lowest common subsumer of $c_{1}$ and $c_{2}$.

The similarity score is $0<\operatorname{Sim}_{\text {wup }}\left(c_{1}, c_{2}\right) \leq 1$ and is never zero since the depth of the lcs is not 0 . And if Simwup $\left(c_{1}, c_{2}\right)=1$, then concepts $c_{1}$ and $c_{2}$ are in the same synset, i.e., similar meanings $\left(c_{1} \equiv c_{2}\right)$ although different words are used.

When the similar pair of concepts was obtained, the OWL property was applied to solve the semantic conflict, for example, owl:equivalentClass or owl:equivalentProperty, as shown in Table 1 and Table 2, respectively.

\subsubsection{Structural Heterogeneity}

Occurs when the same concepts of different systems are modeled with different logical structures. The principle of structural conflict consideration was based on the characteristics of data storing. Although, there are various types of structural heterogeneity, this paper focuses on schematic discrepancies as described below:

- Schematic discrepancies occur when the logical structure of a set of properties and their values belonging to a concept in one system are organized to form a different structure in another system. For example,
Ontology 1 might store or differentiate data in a single class, while Ontology 2 might store the same type of data in Ontology 1 through the property, as shown in the example in Figure 6. It can be seen that the $O 1$ : Photograph class is equivalent to the concept $\mathrm{O}$ : Resource whose property $d c$ :type has the range as the concept $O 2$ : Image.

- In most cases no direct concept to concept mapping is possible. Solution of structural conflicts in this research was achieved through the use of OWL properties, namely, owl:equivalentClass, owl:onProperty, and owl:someValueFrom, as shown in Figure 7.

Table 1. Result of calculation of similarity score between selected classes.

\begin{tabular}{cccc}
\hline Ontology 1 & Ontology 2 & $\begin{array}{c}\text { Similarity } \\
\text { value }\end{array}$ & Similarity structure \\
\hline Object & Resource & 0.625 & owl:equivalentClass \\
Provenance & $\begin{array}{c}\text { Provenance } \\
\text { Statement }\end{array}$ & 1 & owl:equivalentClass \\
Location & Location & 1 & owl:equivalentClass \\
Period & PeriodOfTime & 1 & owl:equivalentClass \\
Period & Period & 1 & owl:equivalentClass \\
Category & SubjectScheme & 0.75 & owl:equivalentClass \\
\hline
\end{tabular}

Table 2. Result of calculation of similarity score between selected properties.

\begin{tabular}{cccc}
\hline Ontology 1 & Ontology 2 & $\begin{array}{c}\text { Similarity } \\
\text { value }\end{array}$ & Similarity structure \\
\hline Name & dc:title & 0.9333 & $\begin{array}{c}\text { owl:equivalent } \\
\text { Property }\end{array}$ \\
Description & dc:description & 1 & $\begin{array}{c}\text { owl:equivalent } \\
\text { Property }\end{array}$ \\
Format & dc:format & 1 & $\begin{array}{c}\text { owl:equivalent } \\
\text { Property }\end{array}$ \\
Added_Date & date & 1 & $\begin{array}{c}\text { owl:equivalent } \\
\text { Property }\end{array}$ \\
& & & owl:equivalent \\
Category & dc:subject & 0.75 & Property \\
& & & 1
\end{tabular}

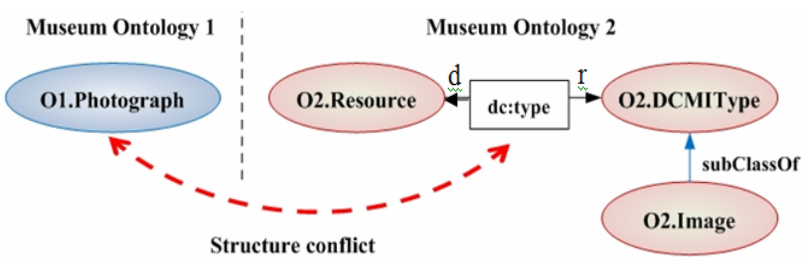

Figure 6. Example of schematic discrepancies. 


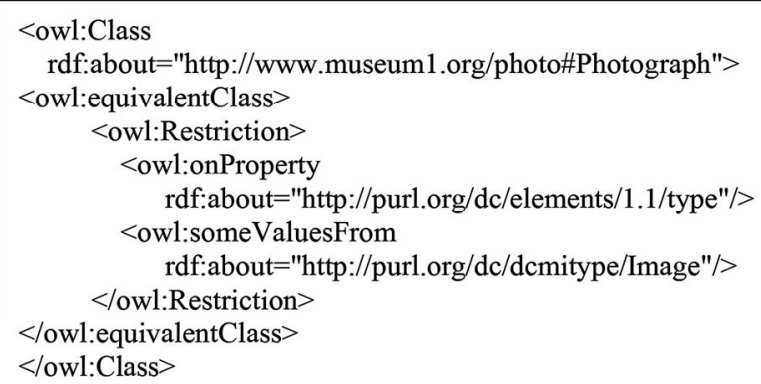

Figure 7. Use of OWL properties to solve the structural conflicts.

\subsection{Research Experiment}

The Ontology-Based Personalized Multimedia Domain derived from ontology mapping of different sources can be used as a core component for semantic data retrieval through the SPARQL as in the following examples:

Example 1 illustrates a query of data from ontology with structural conflicts (as shown in Figure 6). The query in Figure 8 shows a request for the photograph files in the ontology or all instances of the Photograph class.

The results executed from SPARQL command in Figure 8 return all instances of the Photograph class of Ontology 1 and instances from the Resource class in Ontology 2 which has the dc:type values as instances in the Image class. A portion of query results is illustrated in Figure 9.

It can be seen that when the semantic property of OWL is used in the construction and integration of ontologies, semantic retrieval is more efficient. User can use terms defined in one ontology to locate information from one source, but the system is able to retrieve more results from another ontology.

Example 2 illustrates a query of data from ontology according to the users' interest. For this example, Table 3 illustrates the user's interest stored in the user profile ontology (Figure 5) and Table 4 shows details of object files existing in Ontology 1 and Ontology 2.

The query in Figure $\mathbf{1 0}$ shows a request for the object files in the ontology that have format corresponding to the users' interest.

With the benefits of OWL properties in enabling greater inferencing, the museum ontology 1 can define the $O 1$ : format property to be inversed of the 01 : formatOf via the owl:inverse Of property, as shown in Figure 11.

Once the O1:formatOf property is defined to be inverseOf the 01 : format property, user can pose a query by using either 01 :formatOf or $O 1$ format property without creating the instance statement for the $O 1$ :formatOf property.

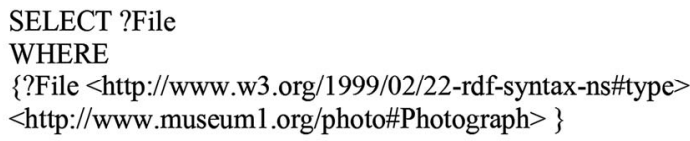

\{?File <http://www.w3.org/1999/02/22-rdf-syntax-ns\#type> $<\mathrm{http}: / /$ www.museuml.org/photo\#Photograph $>$ \}

Figure 8. SPARQL command for retrieving the photograph files.

\begin{tabular}{|l|}
\hline Query Results (10 answers): \\
\hline \multicolumn{1}{|c|}{ File } \\
\hline O1:photograph_01 \\
\hline O1:photograph 04 \\
\hline O2:ResourceImage001 \\
\hline$\underline{\text { O2:ResourceImage } 003}$ \\
\hline$\ldots .$. \\
\hline$\ldots$ \\
\hline
\end{tabular}

Figure 9. Instances results retrieved from the photograph class.

SELECT ?Object

WHERE $\{<$ http://www.UserProfile.org/user\#user_001>

$<$ http://www.UserProfile.org/\#interestInObjectFormat $>$ ?Format .

?Format $<$ http://www.museum1.org/\#formatOf $>$ ?Object \}

Figure 10. SPARQL command for retrieving the object files corresponding to the user's interest.

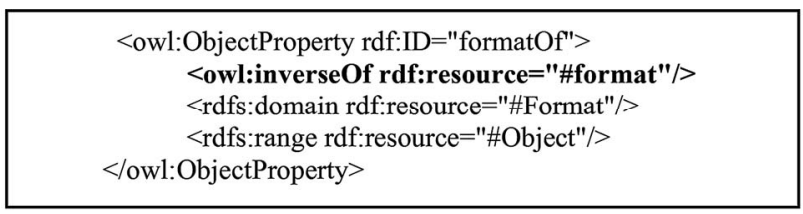

Figure 11. Using owl:inverseOf property.

Table 3. Example of the user's interest stored in the user profile ontology.

\begin{tabular}{cc} 
& User:user_001 \\
Class & Instance \\
InterestPeriod & Renaissance \\
InterestMaterial & gold \\
InterestCategory & art \\
InterestFormat & pdf \\
\hline
\end{tabular}

Table 4. Example of object file description stored in the museum ontology.

\begin{tabular}{ccccc}
\hline Object & Period & Material & Category & Format \\
\hline O1:art_001 & Rebirth & gold & art & pdf \\
O1:art_002 & Rebirth & wood & video & avi \\
O2:art_003 & Renaissance & gold & art & pdf \\
\hline
\end{tabular}


The results executed from SPARQL command in Figure 10 return all instances of the Object class of Ontology 1 and instances from the Resource class in Ontology 2 which was found in the Renaissance period, made from gold, classified in the art category, and has a pdf format. Hence, the object O2:art_003 and O1:art_001 instances that are most matched with the user's interest are returned to a user view.

\subsection{System Evaluation}

This research evaluated the results of experiment and assessed the efficiency of information retrieval from integrated ontologies based on the Precision, Recall and F-Measure [31] values. Precision is the ratio of the number of relevant records retrieved to the total number of irrelevant and relevant records retrieved. Recall is the ratio of the number of relevant records retrieved to the total number of relevant records in the ontology. Precision and Recall values are calculated from Equations (2) and (3) as follows:

$$
\begin{gathered}
\text { Precision }=\frac{A}{A+B} \times 100 \% \\
\text { Recall }=\frac{A}{A+C} \times 100 \%
\end{gathered}
$$

where $A$ is number of relevant records retrieved. $B$ is number of relevant records not retrieved. $C$ is number of irrelevant records retrieved.

Precision and Recall values can be used to calculate F-measure which is defined as a harmonic mean of precision and recall, as shown in Equation (4) below:

$$
F=2 \times\left(\frac{\text { Precision } \times \text { Recall }}{\text { Precision }+ \text { Recall }}\right)
$$

Evaluation of the retrieving efficiency of information interested by 30 users shows that most objects found corresponded to the interest of users. The object information is retrieved on the criterion of classification, format, material, and period of each object with the Precision value of 1.00 the Recall value of 0.93 , and the average of overall efficiency or F-measure was 0.96 . These results were in very high levels.

\section{Conclusions}

This research designed and developed the integration of multimedia information interested by individuals in the museum system based on Semantic Web technology. The multimedia information sources were derived from two museum systems and extraced into ontologies. The researchers developed ontologies with OWL language and integrated the ontologies based on the ontology mapping technique. To solve the semantic heterogeneity, the principle of semantic similarity values via the WordNet is applied to the research. To solve the structural heterogeneity, we employed the OWL properties to be used during the ontology mapping process. In semantic data retrieval, SPARQL language was imperatived for retrieving data in ontologies.

The experiment shows that data retrieving according to 30 users' multiple interests corresponded to their requirements. This was based on the information of each object's period, material, classification, and format, with a Precision value of 1.00 , Recall value of 0.93 , and an average F-measure of 0.96 , indicating high levels of experimental results.

\section{Acknowledgements}

We deeply thank to Department of Computer Science, Faculty of Science, Khon Kaen University for financial support of the research due to research grant: CSKKU12/2554\#8.

\section{REFERENCES}

[1] T. Berners-Lee, J. Hendler and O. Lassila, "The Semantic Web," Scientific American, Vol. 284, No. 5, 2001, pp. 34 43. doi:10.1038/scientificamerican0501-34

[2] D. L. McGuinness and F. van Harmelen, "Owl: Web Ontology Language Overview," 2011. http://www.w3.org/TR/owl-ref/

[3] G. A. Miller, "WordNet: A Lexical Database for English," Communications of the ACM, Vol. 11, No. 38, 1995, pp. 39-41. doi:10.1145/219717.219748

[4] K. Wang, "Improving Engineering Data Management with Semantic Web Techniques," Journal of Service Science and Management, Vol. 1, No. 3, 2008, pp. 199-205. doi:10.4236/jssm.2008.13021

[5] O. Lassila and R. R. Swick, "Resource Description Framework (RDF) Model and Syntax Specification,” 2011. http://www.w3.org/RDF

[6] D. Brickley and R. V. Guha, "Resource Description Framework Schema (RDFS)," 2011. http://www.w3.org/TR/PR-rdf-schema/

[7] G. Karvounarakis, S. Alexaki, V. Christophides, D. Plexousakis and M. Scholl, "RQL: A Declarative Query Language for RDF," Proceedings of World Wide Web, Honolulu, May 7-11, 2002, pp. 592-603.

[8] W3C, "RDQL: A Query Language for RDF," 2011. http://www.w3.org/Submission/RDQL/

[9] E. P. Hommeaux and A. Seaborne, "SPARQL Query Language for RDF," 2011. http://www.w3.org/TR/rdf-sparql-query/

[10] R. Fikes, P. Hayes and I. Horrocks, "OWL-QL: A Language for Deductive Query Answering on the Semantic," Web Semantics: Science, Services and Agents on the World Wide Web, Vol. 2, No. 1, 2004, pp. 19-29. doi:10.1016/S1570-8268(09)00054-7

[11] Dublin Core, "The Dublin Core Metadata Initiative," 2010. http://dublincore.org/ 
[12] D. Beckett, E. Miller and D. Brickley, "Expressing Simple Dublin Core in RDF/XML,” 2010. http://dublincore.org/documents/demes-xml/

[13] S. Kokkelink and R. Schwänzl, "Expressing Qualified Dublin Core in RDF/XML," 2010. http://dublincore.org/documents/dcq-rdf-xml/

[14] J. L. Seng and I. L. Kong, "A Schema and OntologyAided Intelligent Information Integration," Expert Systems with Applications, Vol. 36, No. 7, 2009, pp. 1053810550. doi:10.1016/j.eswa.2009.02.067

[15] W. May and G. Lausen, "A Uniform Framework for Integration of Information from the Web," Information System, Vol. 29, No. 1, 2004, pp. 59-91. doi:10.1016/S0306-4379(03)00005-X

[16] S. Bergamaschi, S. Castano, M. Vincini and D. Beneventano, "Semantic Integration of Heterogeneous Information Sources," Data \& Knowledge Engineering, Vol. 36, No. 3, 2001, pp. 215-249. doi:10.1016/S0169-023X(00)00047-1

[17] S. Zhu and J. Feng, "Using an Ontology to Help Reason about the Information Content of Data," Journal of Software Engineering and Applications, Vol. 3, No. 7, 2010, pp. 629-643. doi:10.4236/jsea.2010.37073

[18] S. Ram and J. Park, "Semantic Conflict Resolution Ontology (SCROL): An Ontology for Detecting and Resolving Data and Schema-Level Semantic Conflicts," IEEE Transactions on Knowledge and Data Engineering, Vol. 16, No. 2, 2004, pp. 189-202. doi:10.1109/TKDE.2004.1269597

[19] D. Sánchez, M. Batet, D. Isern and A. Valls, "OntologyBased Semantic Similarity: A New Feature-Based Approach," Expert Systems with Applications, Vol. 39, No. 9, 2012, pp. 7718-7728. doi:10.1016/j.eswa.2012.01.082

[20] H. Liu, H. Bao and D. Xu, "Concept Vector for Semantic Similarity and Relatedness Based on WordNet Structure," Journal of Systems and Software, Vol. 85, No. 2, 2012, pp. 370-381. doi:10.1016/i.jss.2011.08.029

[21] C. Li and T. W. Ling. "OWL-Based Semantic Conflicts Detection and Resolution for Data Interoperability," Lecture Notes in Computer Science, Springer, New York, 2004, pp. 266-277.
[22] Y. Kalfoglou and M. Chorlemmer, “Ontology Mapping: The State of the Art," The Knowledge Engineering, Vol. 18 , No. 1, 2003, pp. 1-31. doi: $10.1017 / \mathrm{S} 0269888903000651$

[23] D.-N. Chen and Y.-C. Chiang, "Combining Personal Ontology and Collaborative Filtering to Design a Document Recommendation System," Journal of Service Science and Management, Vol. 2, No. 4, 2009, pp. 322-328. doi:10.4236/jssm.2009.24038

[24] X. Aimé, F. Furst, P. Kuntz and F. Trichet, "Prototypicality Gradient and Similarity Measure: A Semiotic-Based Approach Dedicated to Ontology Personalization," Intelligent Information Management, Vol. 2, No. 2, 2010, pp. 65-79. doi:10.4236/iim.2010.22009

[25] B. Solomon, "Using Web Services to Deliver Information Integration," Proceedings of the Museums and the Web Conference, Albuquerque, 22-25 March 2006.

[26] O. Signore, "Ontology Driven Access to Museum Information," Proceedings of the Annual Conference of CIDOC, Zagreb, 24-27 May 2005, pp. 1-8.

[27] E. Sirin, B. Parsia, B. C. Grau, A. Kalyanpur and Y. Katz, "Pellet: A Practical OWL-DL Reasoned," Journal of Web Semantics, Vol. 5, No. 2, 2007, pp. 51-55. doi:10.1016/j.websem.2007.03.004

[28] V. Jalali and A. Bagheri, "Semi-Automated Mapping from RDB to Ontology," Proceedings of the 3rd International Conference on Information \& Knowledge Technology, Mashhad, 27-29 November 2007.

[29] N. Cullot, R. Ghawi and K. Yétongnon, "DB2OWL: A Tool for Automatic Database-to-Ontology Mapping," Proceedings of the 15th Italian Symposium on Advanced Database Systems, Torre Canne, 17-20 June 2007, pp. 491-494.

[30] C. Courtney and M. Rada, "Measuring the Semantic Similarity of Texts," Proceedings of the ACL Workshop on Empirical Modeling of Semantic Equivalence and Entailment, Ann Arbor, 30 June 2005, pp. 13-18.

[31] C. J. van Rijsbergen, "Information Retrieval," Butterworths, London, 1975. 